\title{
Clinicopathological comparison and therapeutic approach to Castleman disease-a case-based review
}

\author{
Małgorzata Wojtyś ${ }^{1}$, Agnieszka Piekarska ${ }^{2}$, Michał Kunc ${ }^{3}$, Konrad Ptaszyński ${ }^{4}$, Wojciech Biernat ${ }^{3}$, \\ Jan Maciej Zaucha ${ }^{2}$, Piotr Waloszczyk ${ }^{5}$, Piotr Lisowski ${ }^{6}$, Bartosz Kubisa ${ }^{1}$, Tomasz Grodzki ${ }^{1}$ \\ ${ }^{1}$ Department of Thoracic Surgery and Transplantation Pomeranian Medical University, Sokołowskiego 11, 70-891 Szczecin, Poland; ${ }^{2}$ Department of \\ Hematology and Transplantology, ${ }^{3}$ Department of Pathomorphology, University Clinical Center, Medical University of Gdańsk, Smoluchowskiego \\ 17, 80-214, Gdańsk, Poland; 'Department of Pathology, Faculty of Medicine, University of Warmia and Mazury, Żołnierska 18, 10-561 Olsztyn, \\ Poland; ${ }^{5}$ Zdunomed Private Health Care Institution, street: Energetyków 2, 70-656 Szczecin, Poland; ${ }^{6}$ Students' Scientific Circle of the Department \\ of Thoracic Surgery and Transplantation, Pomeranian Medical University, Szczecin, Poland \\ Contributions: (I) Conception and design: M Wojtyś; (II) Administrative support: M Wojtyś; (III) Provision of study materials or patients: M Wojtyś, \\ A Piekarska, M Kunc; (IV) Collection and assembly of data: M Wojtyś, A Piekarska, M Kunc, K Ptaszyński, P Waloszczyk; (V) Data analysis and \\ interpretation: M Wojtyś, A Piekarska, M Kunc; (VI) Manuscript writing: All authors; (VII) Final approval of manuscript: All authors. \\ Correspondence to: Małgorzata Wojtyś. Department of Thoracic Surgery and Transplantation Pomeranian Medical University, Sokołowskiego 11, 70- \\ 891 Szczecin, Poland. Email: margaretkaw@wp.pl.
}

\begin{abstract}
Castleman disease (CD) is a rare, B-cell lymphoproliferative disorder affecting lymph nodes and extranodal anatomical locations. Four types of clinical presentations can be distinguished after exclusion of mimics. The first division is into unicentric CD (UCD) and multicentric CD (MCD). MCD is classified further as HHV-8-negative (idiopathic), MCD associated with HHV-8 infection, and POEMS associated MCD. From the histological standpoint, UCD and MCD can be classified as hyaline-vascular (HV), plasma cell (PC), or mixed cellularity (MC) type, with a spectrum of histopathological manifestations. We present clinical and histopathological features and grading of 25 cases of CD classified according to CDCN histological criteria and according to this clinical algorithm, along with outcomes. Here we provide a fineresolution description of the histological features of CD. We review and discuss the current diagnostic algorithm, grading system, and recently recommended treatment options. In the presented group of 25 patients with CD there were 14 women and 11 men in the age range 15-79 years. UCD was identified in 15 patients and it was most often located in mediastinum. MCD most frequently occurred as generalized lymphadenopathy. The most common type of CD was HV. All patients with UCD underwent complete surgical resection with a positive outcome. Patients with MCD had diagnostic partial surgical excision of the lesions, later followed by different types of treatment (corticosteroids, chemotherapy, radiotherapy, immunomodulatory agents) or 'watch and wait'. In four cases CD was associated with other malignancies (laryngeal cancer, small lymphocytic lymphoma, gallbladder cancer with hepatic metastases, primary squamous cell lung cancer). The accuracy of histopathological examination is essential and re-evaluation has to be performed in case of relapse or unexpected course of CD. Treatment tailored to fit the disease type and severity should follow the novel recommendations, including anti-IL-6 treatment in the case of MCD.
\end{abstract}

Keywords: Castleman disease (CD); lymphadenopathy; mediastinal tumor; benign lung lesion

Submitted Sep 17, 2019. Accepted for publication Oct 21, 2019.

doi: $10.21037 /$ jtd.2019.10.73

View this article at: http://dx.doi.org/10.21037/jtd.2019.10.73

(c) Journal of Thoracic Disease. All rights reserved. 


\section{Introduction}

Castleman's disease (CD), also known as giant lymph node hyperplasia or angiofollicular lymphoid hyperplasia, is a benign lymphoproliferative disorder affecting both lymph nodes and extranodal loci $(1,2)$. With an occurrence rate of 21 to 25 cases per million person-years, it is classified as an orphan disease. In clinical practice, two variants exist: unicentric CD (UCD; 75\%) and multicentric CD (MCD; $\sim 25 \%$ ), the latter with various clinical manifestations $(2,3)$. Both can be classified into one of the three main histological types: hyaline-vascular (HV), plasma cell (PC), or mixed. A plasmablastic subtype is observed in $\mathrm{HHV}-8$-positive patients. CD can occur in practically any part of the body, but arises predominantly in the thorax $(\sim 70 \%)$, followed by the abdomen and pelvis $(\sim 15 \%)$ and neck $(\sim 15 \%)(2,4,5)$. Despite numerous case presentations since Castleman et al. first report in 1954, CD remains a diagnostic and therapeutic challenge (6). Histopathological examination remains mandatory for definitive diagnosis. UCD in particular is often an unexpected discovery during routine examinations. In contrast, MCD can manifest with a very serious hypercytokinemia-driven inflammatory syndrome mostly caused by interleukin IL-6 (7).

In this study, we present 25 cases involving CD patients and the associated histopathological and clinical manifestations (8). The case presentation aims to illustrate the utility and adequacy of current diagnostic criteria and treatment options recommended by the Castleman Disease Collaborative Network (CDCN) (8). We also provide a short review of recommendations.

\section{Methods}

\section{Patients}

We retrospectively analyzed histopathological data for all consecutive patients diagnosed with CD from 2002 to 2018 in two university centers (Medical University of Gdansk and Pomeranian Medical University of Szczecin). The clinical data were gathered retrospectively from medical records. Informed patient's consent was obtained.

\section{Patbology}

Diagnosis of CD subtype was established by experienced pathologists and revised once more for confirmation including all staining (hematoxylin \& eosin and immunohistochemical). Moreover, in MCD cases, we applied a grading system proposed by CDCN (8). Additional staining including latency-associated nuclear antigen (LANA)-1 was performed to identify HHV-8positive cases.

\section{Histopathological features of CD}

CD involves a spectrum of histopathological manifestations. In the HV subtype, follicles are usually enlarged, hypervascular, and hyalinized. For an inexperienced pathologist, this picture represents a potential diagnostic pitfall, leading to misdiagnosis of thymoma in cases of mediastinal masses or ectopic spleen in abdominal CD (9). Follicular hyperplasia in $\mathrm{CD}$ is accompanied by a regressive transformation of germinal centers, characterized by a paucity of lymphocytes that have been replaced by abundant dysplastic follicular dendritic cells (FDCs), hyaline material, and prominent sclerotic vessels. Lymphocytes comprising mantle zone form concentric rimming around the follicles, leading to an "onion-skin" appearance (10). The combination of follicle-penetrating hyalinized vessels originating from the paracortex and an expanded mantle zone are referred to as the "lollipop sign".

Interfollicular areas contain multiple postcapillary high endothelial venules with obliterated sinuses and consist of plasmacytoid dendritic cells, TdT-positive T lymphocytes, eosinophils, and plasma cells. Tight aggregates of CD123+ plasmacytoid dendritic cells are highly sensitive markers of CD (11).

The prevalence of follicular hyperplasia or expansion of interfollicular areas may subclassify CD into the follicular type and the stroma-rich type, which is important in the differential diagnosis process. In rare cases of stromarich type $\mathrm{CD}$, especially in the retroperitoneum, there is a proliferation of vasculature and actin-positive myoid cells (angiomyoid proliferative lesions) (12). Excessive proliferation of FDCs followed by dysplasia can lead to malignant transformation into follicular dendritic cell sarcoma (13).

In the PC subtype, crucial diagnostic features can be seen in interfollicular areas, which are covered with sheets of polytypic plasma cells. Lymphoid follicles typically show hyperplastic germinal centers. MCD frequently presents with a mixture of $\mathrm{HV}$ and $\mathrm{PC}$ types, and the recent $\mathrm{CDCN}$ group consensus included a dedicated grading system to classify MCD into hypervascular, PC, or mixed histology. Table 1 presents the differential diagnoses and diagnostic pitfalls of both HV and PC CD (14-17). 
Table 1 Differential diagnosis of CD.

\begin{tabular}{|c|c|}
\hline Feature & Differential diagnosis \\
\hline \multicolumn{2}{|l|}{ Hyaline-vascular type } \\
\hline Nodular architecture & Follicular lymphoma \\
\hline \multirow{4}{*}{$\begin{array}{l}\text { Regressively } \\
\text { transformed germinal } \\
\text { centers }\end{array}$} & HIV infection \\
\hline & Corticosteroid treatment \\
\hline & Organ transplant recipients \\
\hline & Angioimmunoblastic T-cell lymphoma \\
\hline \multirow{3}{*}{$\begin{array}{l}\text { Mantle zone } \\
\text { expansion }\end{array}$} & Mantle zone hyperplasia \\
\hline & $\begin{array}{l}\text { Mantle zone pattern of mantle cell } \\
\text { lymphoma }\end{array}$ \\
\hline & $\begin{array}{l}\text { Monocytoid B-cell pattern in nodal } \\
\text { marginal zone lymphoma }\end{array}$ \\
\hline \multirow{4}{*}{$\begin{array}{l}\text { Plasmacytoid } \\
\text { dendritic cells }\end{array}$} & Kikuchi necrotizing lymphadenitis \\
\hline & Chronic myelomonocytic leukemia \\
\hline & Classical Hodgkin lymphoma \\
\hline & Autoimmune diseases \\
\hline \multirow[t]{4}{*}{$\begin{array}{l}\text { Hypervascularity and/ } \\
\text { or hyalinization }\end{array}$} & $\begin{array}{l}\text { Various B-cell lymphomas (follicular } \\
\text { lymphoma, mantle zone lymphoma, } \\
\text { nodal marginal zone lymphoma) }\end{array}$ \\
\hline & Rheumatoid arthritis \\
\hline & HIV infection \\
\hline & Thymoma \\
\hline \multicolumn{2}{|l|}{ Plasma cell type } \\
\hline \multirow{7}{*}{$\begin{array}{l}\text { Abundant } \\
\text { interfollicular plasma } \\
\text { cells }\end{array}$} & Lymphoplasmacytic lymphoma \\
\hline & Plasmacytoma \\
\hline & Plasmablastic lymphoma \\
\hline & IgG4-related diseases \\
\hline & Rheumatoid arthritis \\
\hline & Rosai-Dorfman disease \\
\hline & $\begin{array}{l}\text { Cytomegalovirus or Epstein-Barr virus } \\
\text { infection }\end{array}$ \\
\hline \multirow[t]{2}{*}{ Hyperplastic follicles } & Reactive follicular hyperplasia \\
\hline & Follicular lymphoma \\
\hline
\end{tabular}

\section{Diagnostic criteria for $C D$}

The CD spectrum includes several distinct diseases that were evaluated by an international group of experts affiliated with the CDCN. Members of the working group have published diagnostic criteria to help pathologists and clinicians distinguish CD from CD-mimicking pathologies, identify the CD type, and assess its severity $(8,18-20)$.

After identification of CD-like features on histopathological examination or resected tissue, the patient's condition should be classified into one of the distinguishable types of $\mathrm{CD}$. The first step is to exclude diseases that can lead to Castleman-like features on histopathology and mimic CD. The following pathologies must be ruled out: infection-related disorders [EpsteinBarr virus (EBV)-related mononucleosis or chronic EBV infection, inflammation and lymphadenopathy caused by cytomegalovirus, toxoplasmosis, active tuberculosis], autoimmune diseases (rheumatoid arthritis, systemic lupus erythematosus, Still disease, juvenile idiopathic arthritis, autoimmune lymphoproliferative syndrome) and malignant/ lymphoproliferative disorders (Hodgkin lymphoma, nonHodgkin lymphoma, multiple myeloma, primary lymph node plasmacytoma, follicular dendritic cell sarcoma) (8).

The second step is to assess the extent of the disease. Staging should be preferably performed with PET/CT (positron emission tomography/computed tomography), but CT is acceptable if access to PET/CT is difficult (21). PET-CT also enables identification of lymphoma or other malignancy $(22,23)$.

The next step is to test for HHV-8 and HIV infection, and ultimately to perform laboratory tests. The basic requirements include complete blood count, creatinine, C-reactive protein, and/or erythrocyte sedimentation rate, albumin, serum protein electrophoresis, and immunoglobulin (Ig) G level. Other tests that are useful for assessing disease severity in cases of MCD include IL-6, soluble IL-2 receptor, vascular endothelial growth factor (VEGF), lactate dehydrogenase, IgA, IgM, and B2microglobulin (8). The basic features of the four types of $\mathrm{CD}$ are presented in Table 2.

\section{UCD}

Unicentric disease affects a single lymph node station. Patients are usually HIV-negative and no systemic symptoms or laboratory findings will reflect increased IL-6 (24).

\section{MCD}

MCD affects more than one lymph node station and often manifests as generalized lymphadenopathy with an enlarged spleen or liver. MCD is subdivided into HHV-8positive MCD, POEMS (polyneuropathy, organomegaly, endocrinopathy, monoclonal proteins, and skin changes)related $M C D$, and idiopathic MCD (iMCD). The 
Table 2 Basic characteristics of different types of Castleman disease

\begin{tabular}{|c|c|c|c|c|}
\hline Type of CD & Lymphadenopathy & Pathology & $\begin{array}{l}\text { IL-6-driven inflammatory } \\
\text { syndrome* }\end{array}$ & Virologic status \\
\hline Unicentric & $\begin{array}{l}\text { Localized (single mass or } \\
\text { single lymph node station) }\end{array}$ & $90 \% \mathrm{HV}$ variant & Typically not & $\begin{array}{l}\text { Negative for HHV-8 by Q-PCR or } \\
\text { negative LANA-1 stain }\end{array}$ \\
\hline $\begin{array}{l}\text { Multicentric HHV-8- } \\
\text { positive }\end{array}$ & $\begin{array}{l}\text { Generalized +/- } \\
\text { hepatosplenomegaly }\end{array}$ & $\mathrm{PC}$ or $\mathrm{PB}$ variant & Yes & $\begin{array}{l}\text { Positive for HHV-8 by Q-PCR; } \\
\text { possible HIV-positivity }\end{array}$ \\
\hline $\begin{array}{l}\text { POEMS-associated } \\
\text { MCD }\end{array}$ & $\begin{array}{l}\text { Generalized +/- } \\
\text { hepatosplenomegaly }\end{array}$ & $\mathrm{PC}$ variant & $\begin{array}{l}\text { Typically IL-6 and VEGF- } \\
\text { driven }^{\star \star \star}\end{array}$ & $\begin{array}{l}\text { Negative for HHV-8 by Q-PCR or } \\
\text { negative LANA- } 1 \text { stain }\end{array}$ \\
\hline
\end{tabular}

${ }^{*} B$-symptoms: fever, night sweats, weight loss, fatigue; possible laboratory findings: anemia, thrombocytopenia or thrombocytosis, elevated acute phase proteins, C-reactive protein, fibrinogen, ferritin, elevated erythrocyte sedimentation rate, polyclonal hypergammaglobulinemia, abnormal kidney function, increased IL-6, VEGF, IL-1, IL-10. **, thrombocytopenia, anasarca, fever, reticulin fibrosis of bone marrow, renal failure, organomegaly. ${ }^{* *}$, polyneuropathy, organomegaly, endocrinopathy, monoclonal protein, skin changes. CD, Castleman disease; IL-6, interleukin-6; VEGF, vascular endothelial growth factor; HHV, human herpesvirus; HIV, human immunodeficiency virus; HV, hyaline vascular; PC, plasma cell; PB, plasmablast; MC, mixed cellularity; Q-PCR, quantitative polymerase chain reaction; LANA-1, Latency-associated nuclear antigen.

symptomatology of HHV-8-positive MCD is related to excessive release of viral IL-6, while iMCD is driven by proinflammatory, mostly IL-6 hypercytokinemia. They can potentially be distinguished through detection of HHV-8 LANA-1 on immunohistochemical staining and/or positive results of virus replication in molecular examinations (quantitative PCR; Q-PCR) of peripheral blood. As its name implies, POEMS-associated MCD manifests with polyneuropathy, organomegaly, endocrinopathy, monoclonal proteins, and skin lesions. The symptomatology of a recently identified clinical subtype of iMCD designated as TAFRO consists of thrombocytopenia, anasarca, fever, reticulin fibrosis/renal failure, and organomegaly (25).

\section{HHV-8-positive MCD}

HHV-8 infects plasmablasts and B lymphocytes. Virusderived IL-6 can directly stimulate the IL-6 receptor without co-stimulation. Diagnosis is based on characteristic histopathology of excised lymphoid tissue and detection of actively replicating HHV-8 and/or a positive LANA-1 stain. In case of HIV positivity, patients should be also screened for the secondary malignancies such as Kaposi's sarcoma and $\mathrm{HIV}$-associated lymphomas (26,27).

\section{HHV-8-negative MCD (iMCD)}

The reason for IL-6 hypercytokinemia can be rarely identified (28). The clinical manifestation can be determined by polymorphism of the IL- 6 receptor, IL-6 release by malignant cells, an unknown virus, or germline mutation of immune cells. According to international, evidence-based CDCN consensus, both one major and a minimum of two of eleven minor criteria with at least one laboratory abnormality are required to diagnose iMCD (8). The major criteria include histopathologic lymph node features consistent with the iMCD spectrum and enlarged lymph nodes ( $\geq 1 \mathrm{~cm}$ in the short-axis diameter) in $\geq 2$ lymph node stations. The minor laboratory criteria include elevated CRP $(>10 \mathrm{mg} / \mathrm{L})$ or ESR $(>15 \mathrm{~mm} / \mathrm{h})$, anemia (hemoglobin $<12.5 \mathrm{~g} / \mathrm{dL}$ for males, $<11.5 \mathrm{~g} / \mathrm{dL}$ for females), thrombocytopenia (platelets $<150 \mathrm{G} / \mathrm{L}$ ) or thrombocytosis (platelets $>400 \mathrm{G} / \mathrm{L}$ ), hypoalbuminemia (albumin $<35 \mathrm{~g} / \mathrm{L}$ ), renal dysfunction estimate glomerular filtration rate $\left(\mathrm{eGFR}<60 \mathrm{~mL} / \mathrm{min} / 1.73 \mathrm{~m}^{2}\right.$ ) or proteinuria (total protein $150 \mathrm{mg} / 24 \mathrm{~h}$ or $10 \mathrm{mg} / 100 \mathrm{~mL}$ ), and polyclonal hypergammaglobulinemia (total gamma-globulin or IgG $>17 \mathrm{~g} / \mathrm{L})$. Clinical abnormalities listed as minor criteria include constitutional symptoms (night sweats, fever $>38$ degrees $\mathrm{C}$, weight loss or fatigue), large spleen and/or liver, fluid accumulation (edema, anasarca, ascites, or pleural effusion), eruptive cherry hemangiomatosis or violaceous papules, and lymphocytic interstitial pneumonitis. Infectionrelated disorders, autoimmune or autoinflammatory diseases, and malignant/lymphoproliferative disorders must be excluded. Detection of autoimmune antibodies is not exclusionary (8). In the case of TAFRO syndrome, bone marrow histopathological examination should be performed. 


\section{POEMS-associated MCD}

POEMS-associated MCD is believed to derive from increased IL-6 and VEGF production by monoclonal plasma cells leading to clinical symptomatology. In particular, VEGF correlates with disease activity. Most POEMS are lambda chain restricted $(29,30)$. Bone marrow histopathological examination with a search for monoclonal plasma cells is required. There is also a POEMS syndrome without evidence of a clonal plasma cell disorder (31).

\section{Therapeutic approach of $C D$}

The choice of appropriate therapy for CD depends on careful assessment of disease, localization, clinical symptoms, and biochemical and immunological markers, but first and foremost, it depends on histological features. Available treatment options include surgical excision or debulking; corticosteroids; immunotherapy with siltuximab, tocilizumab, rituximab, or anakinra; chemotherapy with etoposide, cladribine, or cyclophosphamide; various combination immunochemotherapeutic protocols; and immunomodulatory/immunosuppressive treatment with thalidomide, sirolimus, bortezomib, or cyclosporin A $(18,19)$.

\section{UCD}

UCD usually presents as solitary lymphoid tissue overgrowth or lymphadenopathy that is curable with surgical extirpation. It might be asymptomatic or lead to compression-related symptoms. In case of an unresectable mass or a dangerous localization, remission can be achieved or the size can be reduced with administration of corticosteroids and/or rituximab, and a delayed surgical intervention may be offered afterwards. A reported role of radiotherapy should not be omitted $(19,32)$.

When disease relapses, the excised lymphoid mass should be carefully examined by the pathologist because it may contain foci of lymphoma transformation. In such cases, a patient requires lymphoma-like treatment with a combination immunochemotherapy, e.g., R-COP or R-CHOP (rituximab, cyclophosphamide, doxorubicin, vincristine, prednisone).

\section{MCD}

\section{HHV-8-positive MCD}

Treatment with rituximab is effective because it depletes the viral reservoir and reduces the risk of lymphoma (33). Some patients may additionally require etoposide. The utility of tocilizumab remains under evaluation in clinical trials (34).
Siltuximab does not bind to viral IL-6, as has been established in preclinical trials. Some experts recommend maintenance therapy with valganciclovir to control viral replication. HIV-positive patients with HHV-8-positive MCD must be simultaneously administered adequate antiretroviral therapy (26). iMCD

Historically iMCD was treated with corticosteroids, rituximab, and chemotherapeutics from the CHOP regimen. According to novel recommendations of $\mathrm{CDCN}$, therapy for iMCD should be preceded by stratification to a non-severe or severe clinical manifestation. The criteria for severe iMCD include Eastern Cooperative Oncology Group score $\geq 2$, kidney failure stage IV (estimated glomerular filtration rate $<30 \mathrm{~mL} / \mathrm{min} / 1.73 \mathrm{~m}^{2}$; creatinine $>3.0 \mathrm{mg} / \mathrm{dL}$ ), effects of hypercytokinemia and hypoalbuminemia such as anasarca and/or ascites and/or pleural effusion, anemia with hemoglobin concentration $\leq 8 \mathrm{~g} / \mathrm{dL}$, and pulmonary involvement (interstitial pneumonitis, dyspnea) (18).

Therapy for non-severe iMCD should target IL-6. Siltuximab, a monoclonal antibody to IL-6, is a recommended front-line option with a $34 \%$ response rate demonstrated in clinical trials and already approved by the US Food and Drug Administration and European Medicines Agency (35). Tocilizumab, which blocks the IL-6 receptor, is effective as monotherapy or in combination therapy in more serious cases $(19,30)$. Both tocilizumab and siltuximab are well-tolerated. Tocilizumab can be used, if siltuximab is not available or approved (18). In case of the oligosymptomatic form with a low IL-6 concentration, treatment that blocks IL-6 may be less effective, and rituximab with steroids should be administered. Some patients respond to anti-IL-1 therapy. Immunomodulatory/ immunosuppressive agents are considered second- or thirdline therapy, and their administration should be initiated in consultation with a specialist (30).

In severe iMCD with organ failure, patients must be closely monitored, and immuno-chemotherapy should be considered, if no response occurs after one week of therapy with siltuximab or whenever the clinical situation deteriorates. Recommended chemotherapeutic protocols include R-CHOP, R-VTD-PACE (rituximab, bortezomib, dexamethasone, thalidomide, cisplatin, doxorubicin, cyclophosphamide, etoposide), or less toxic rituximab with etoposide and cyclophosphamide (18). Tocilizumab can be used instead of siltuximab.

\section{POEMS-associated MCD}

Treatment should target the monoclonal plasma cell population. For localized manifestation (an isolated 

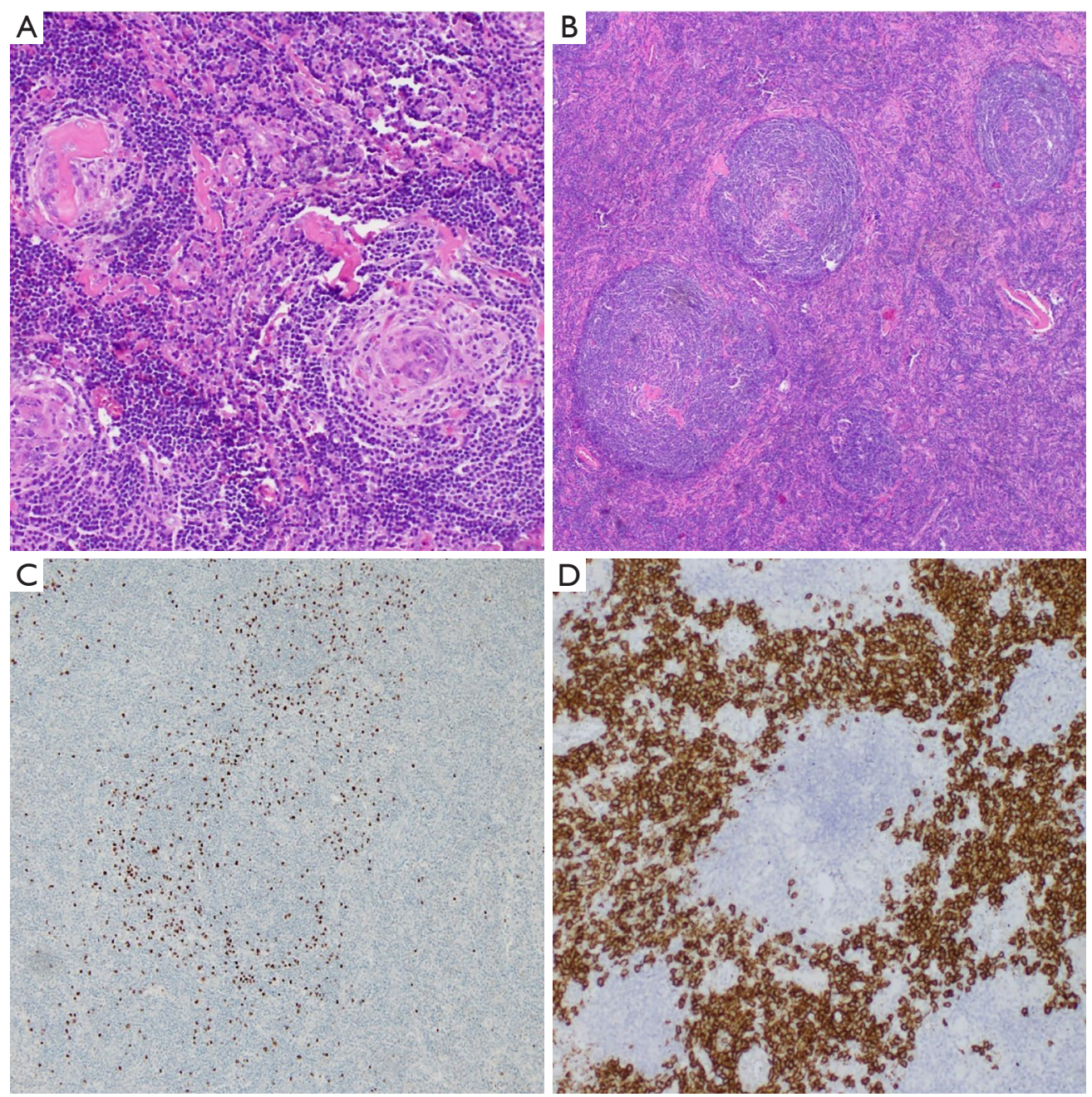

Figure 1 The histologic view of different forms of Castleman disease (CD): the hyaline vascular type (A,B) vs. plasma cell type (C,D). Hyaline vascular type of CD: (A) lymphoid follicles with atrophic germinal centers showing proliferation of follicular dendritic cells. Hyalinized vessels radially penetrate germinal centers (lollipop sign) (HE, ×100); (B) lymphoid follicles with atrophic germinal centers and thickened mantle zones (onion skin appearance) (HE, $\times 40)$. Plasma cell type of CD: (C) scattered cells show LANA-1 antigen expression suggesting HHV-8 virus infection and the mixed cellularity type of CD (HE, ×40); (D) polyclonal, CD138-positive plasma cell proliferation in the interfollicular zone $(\mathrm{HE}, \times 100)$.

plasmacytoma), radiation therapy is often efficient. For disseminated disease, high-dose melphalan with autologous peripheral hematopoietic cell transplantation is the most effective therapeutic option (31).

\section{Results}

\section{Patient characteristics}

Among analyzed patients, 15 (60\%) presented hyaline vascular morphology on histology (Figure 1A,B), 8
(32\%) presented plasma cell type form (Figure 1C,D), and $2(8 \%)$ presented the mixed form (Figure $2 A, B, C)$. Unicentric localization including a single lesion and single lymph node station (Figure 3) was diagnosed in 15 (60\%) patients, including 13 cases with $\mathrm{HV}$ and 2 cases with PC morphology (mesentery tumor and relapsing cervical lymphadenopathy). Among the remaining ten cases of MCD, only two involved HV morphology (Figure 4), and two presented the mixed type of CD (Figure 5). HHV8 -positive MCD was diagnosed in only one case. Table 3 presents detailed patient characteristics. 

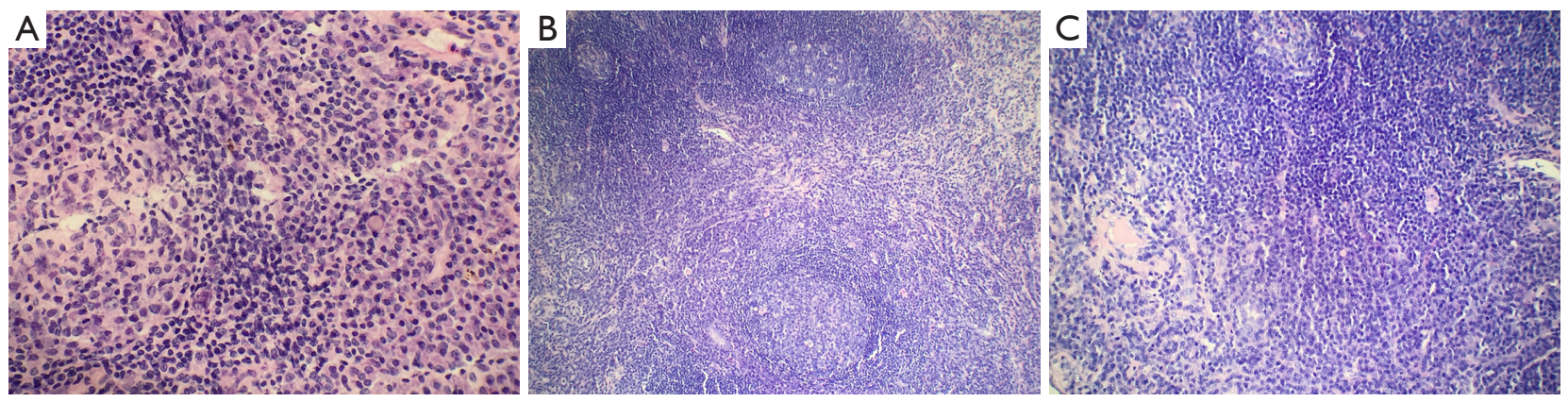

Figure 2 Histopathology of the lung lesion with features of a mixed variant of Castleman disease. (A) Close-up view of the lesion with a germinal center and plasma cells in the interfollicular area (HE, $\times 400)$; (B) low-power view of the lesion showing hyperplastic germinal centers, broad mantle zone, and hypervascularity of the interfollicular area where plasma cells also can be seen $(\mathrm{HE}, \times 100)$; $(\mathrm{C})$ one germinal center with hyperplasia and another with regression and a hyaline change $(\mathrm{HE}, \times 200)$.

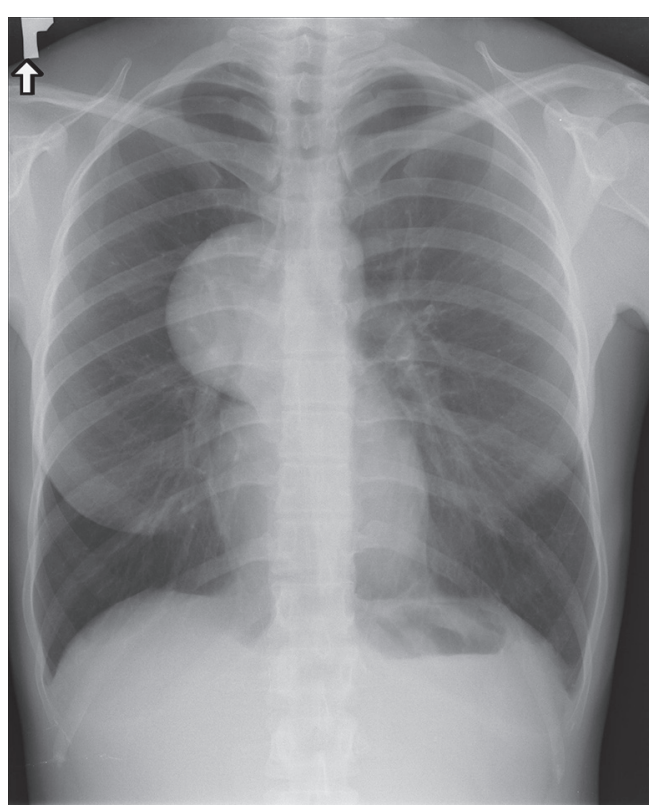

Figure $3 \mathrm{X}$-ray shows a tumor size of $90 \mathrm{~mm} \times 60 \mathrm{~mm}$ in the area of the right pulmonary hilum.

\section{Histopathological details}

Figure 6 shows a graphic representation of Castleman Disease Collaborative Network (CDCN) histological grading system in our cohort. Although this system is dedicated to iMCD, it summarizes the most important features of both the HV and PC types of CD and thus can be used to clearly present the microscopic patterns that prevailed in this case group. In the vast majority of UCD cases, three combined features occurred: regression of germinal centers, prominent FDCs, and hypervascularity,

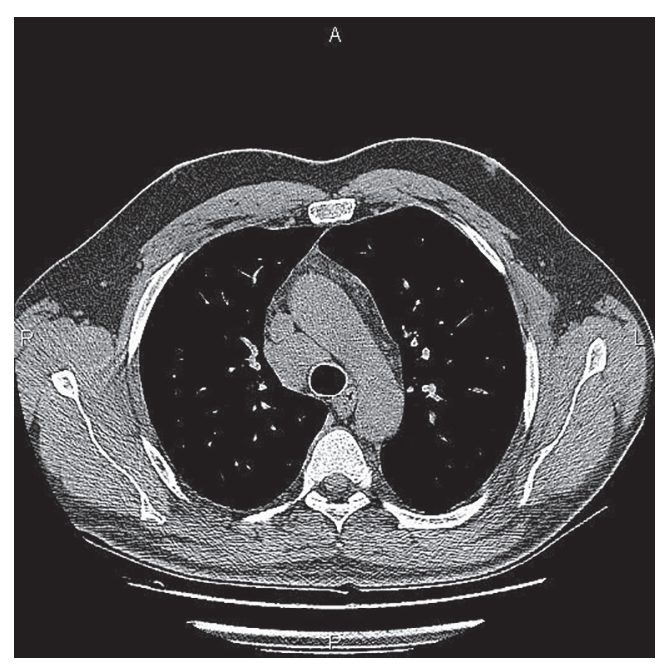

Figure 4 Chest CT shows a tumor of $30 \mathrm{~mm} \times 39 \mathrm{~mm} \times 54 \mathrm{~mm}$ in the middle mediastinum. There also are enlarged lymph nodes in the upper mediastinum.

which is consistent with an HV type diagnosis. Moreover, nine cases $(60 \%)$ of UCD presented with germinal center "twinning", 8 (53.3\%) had the "lollipop" sign, and 11 $(73.3 \%)$ showed "onion skin" mantle zones. Interfollicular plasmacytosis was identified only in $5(33.3 \%)$ cases.

In contrast, most MCD cases $(9 / 10,90 \%)$ had at least grade 2 plasmacytosis. They also showed frequent regression of germinal centers with FDC proliferation and hypervascularity. Hyperplastic germinal centers were observed in $7 / 10$ cases of MCD (70\%), but they were not prominent (low grading scores) and in one case of UCD. The relatively low prominence of germinal center hyperplasia is consistent with other studies (8). 

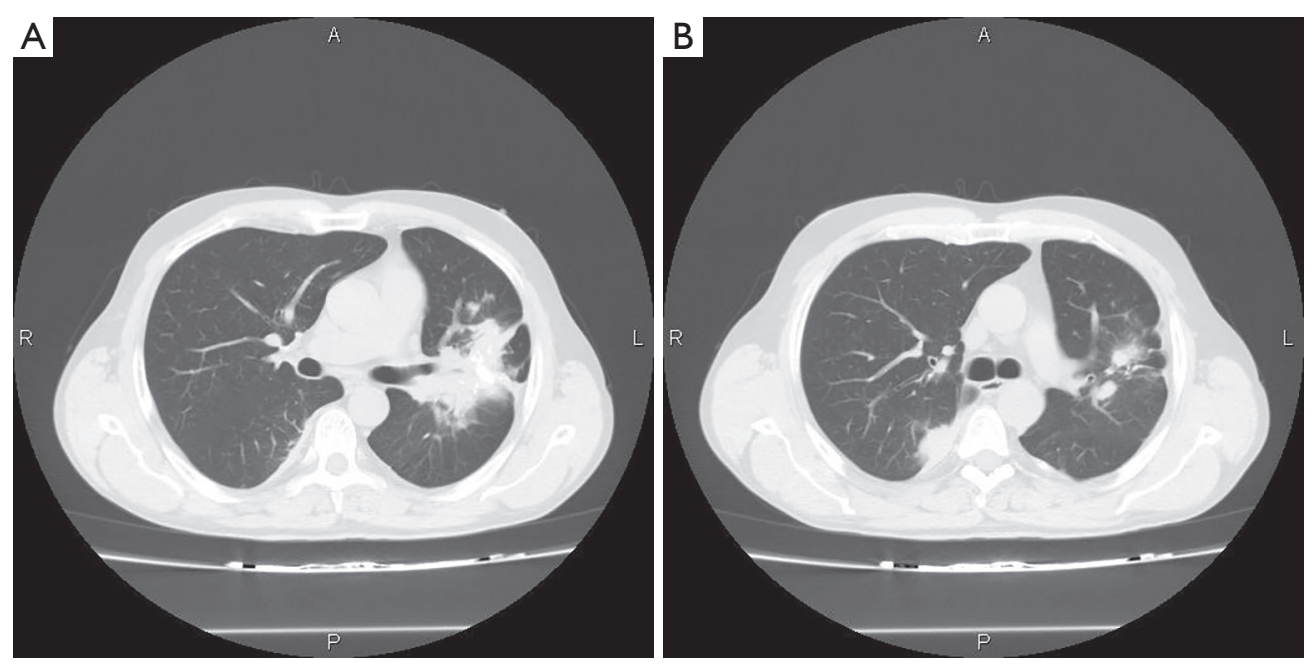

Figure 5 Chest CT shows an irregular infiltration of $7 \mathrm{~cm} \times 5 \mathrm{~cm}$ in the left lung. An infiltration of $4 \mathrm{~cm} \times 2 \mathrm{~cm}$ can also be seen in the right lung near the vertebral column.

\section{Medical procedures implemented in presented patients and follow-up}

The duration of follow-up in the presented group of 25 patients with CD ranged from 1 to 228 months. Summarized clinical data are presented in Table 3.

\section{UCD}

In the UCD group of 15 patients, complete surgical excision was the therapeutic choice with excellent long-term outcomes. In five patients with mediastinal involvement, a surgical approach consisted of videomediastinoscopy (two cases), videothoracoscopy (one case), thoracotomy (two cases). Four patients with cervical lymphadenopathy and one patient with axillary lymphadenopathy underwent surgical excision of lymph nodes. Four patients had laparotomy and surgical excision of the tumor (two cases of retroperitoneal tumors, one case with a tumor of the stomach area, one case with mesentery tumor). In one patient with parapharyngeal tumor surgical excision of the lesion was complemented by local radiotherapy (Rtx).

One patient with cervical lymphadenopathy suffered from laryngeal cancer treated with Rtx and one patient with mediastinal tumor had additionally small lymphocytic lymphoma treated with chemotherapy (4 courses of fludarabine with cyclophosphamide-Cy).

Fourteen patients in this group are still alive. Patient with a tumor of the stomach area died in the course of disseminated cancer (gallbladder cancer with hepatic metastases).

\section{MCD}

In the presented group, there were 10 patients with MCD. Four patients with generalized lymphadenopathy underwent lymph node excision under local analgesia (three cases) or laparotomy with lymph node excision (one case). One patient achieved regression after additional therapy with thalidomide, cyclophosphamide (Cy) and prednisone.

Thalidomide was administered to one patient as the first line treatment with a primarily good response. Relapse was associated with severe hypercytokinemia symptoms that progressed to hemophagocytic lymphohistiocytosis (HLH) and, as there was no access to anti-IL6 agents, sequential therapy with cladribine, $\mathrm{Cy}$ and etoposide, cyclosporine (CsA), dexamethasone (Dexa) was administered without success.

One patient who underwent laparotomy was successfully treated with prednisone. One patient underwent repetitive surgical excisions of axillary and inguinal lymph nodes, which were complemented by local Rtx and chemotherapy (Ctx).

There were three patients with isolated lung involvement of MCD. Two of them experienced explorative thoracotomy, and one had thoracotomy with upper lobectomy, mediastinal lymphadenectomy and excision of the tumor of the right lower lobe. They remain in stable clinical condition without any further therapy.

Out of two patients with mediastinal multifocal lymphadenopathy, one underwent videomediastinoscopy and is being treated with thalidomide with regression 


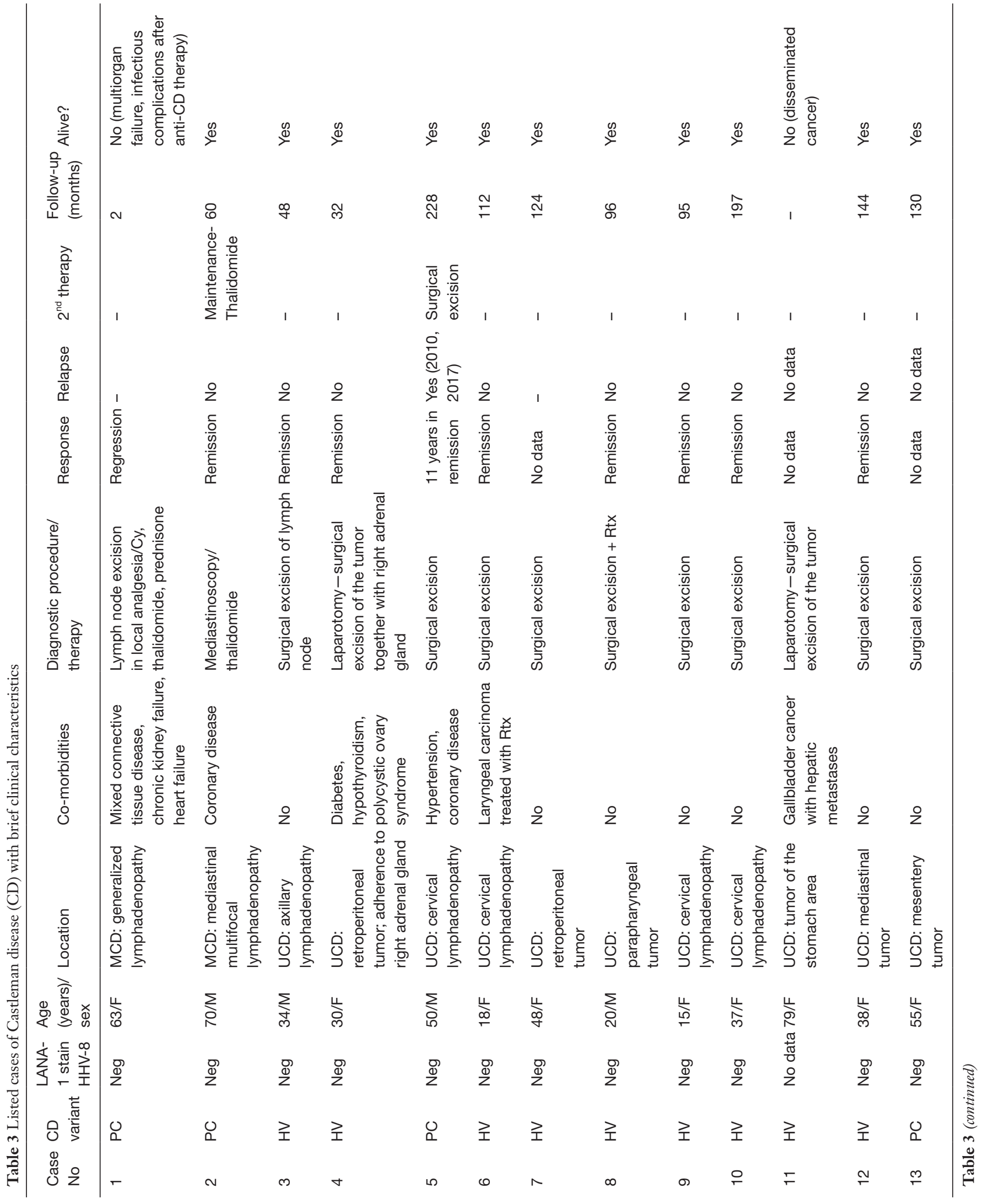




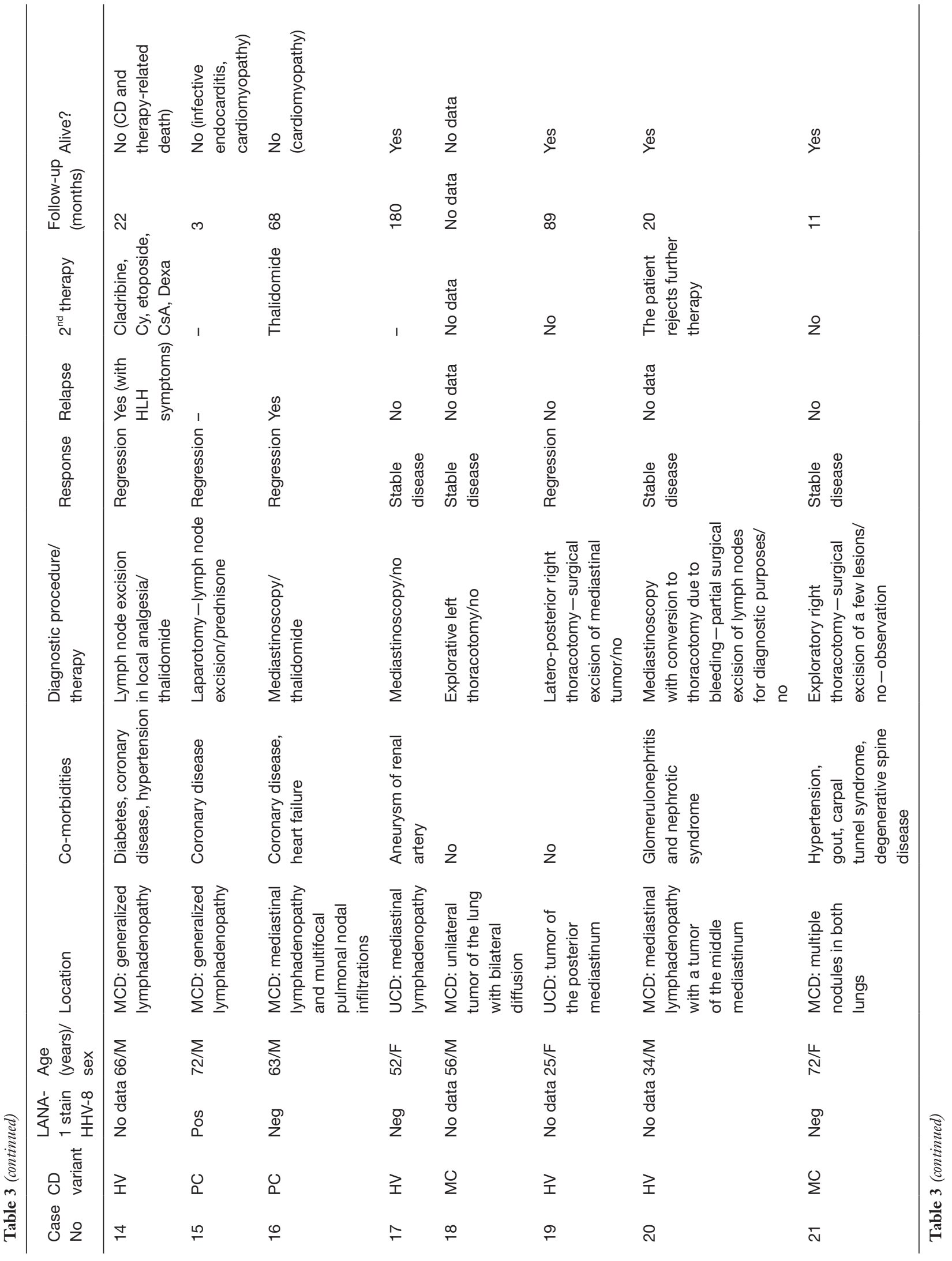




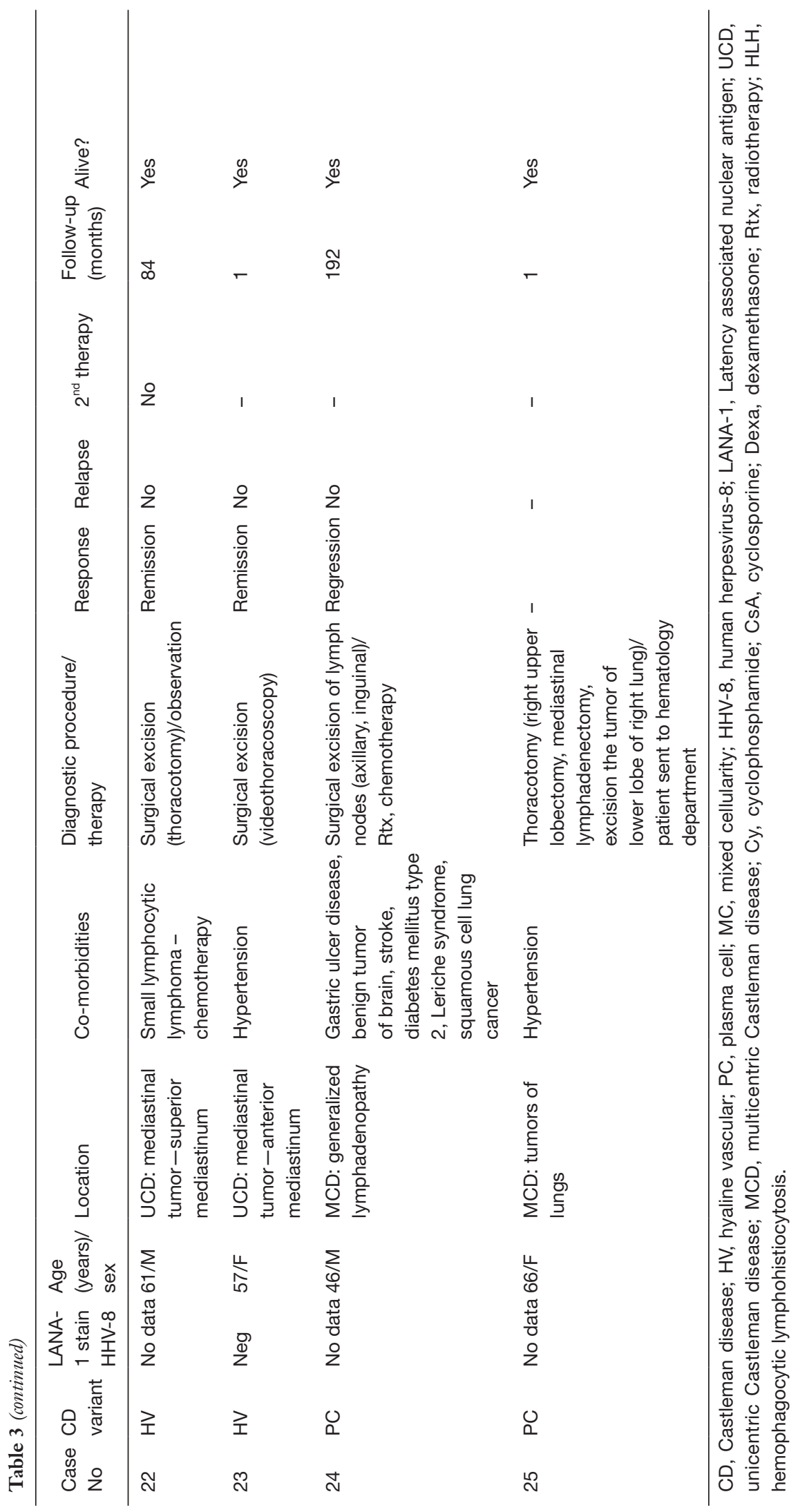




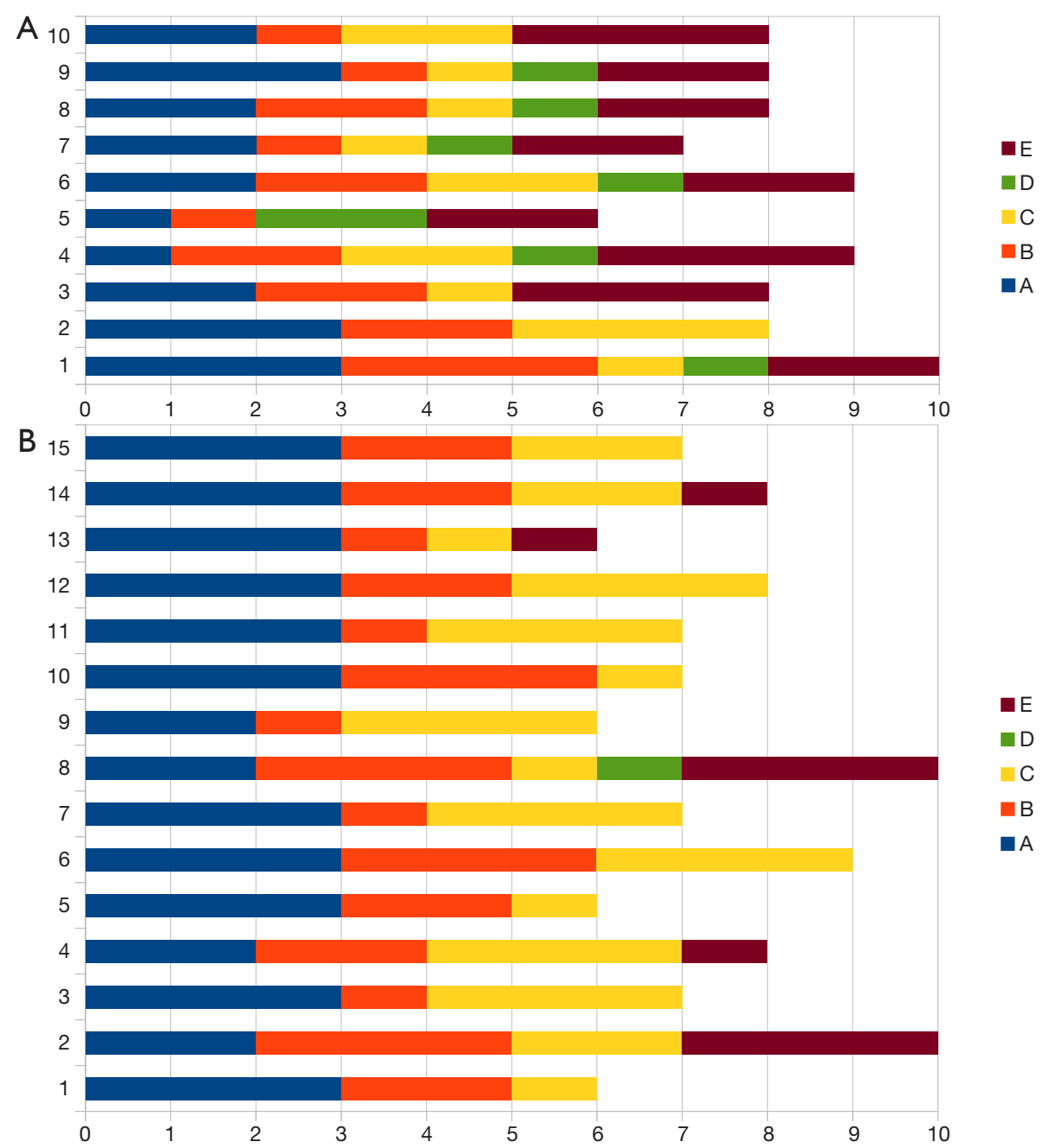

Figure 6 Graphic representation of Castleman Disease Collaborative Network (CDCN) histological grading system in our cohort. (A) Multicentric Castleman disease (MCD) grading; (B) unicentric Castleman disease (UCD) grading; (C) key.

of the lesions. In another one (HV type of MCD) videomediastinoscopy must have been converted to thoracotomy due to intraoperative bleeding. The tumor had hypervascular features, and despite careful dissection of tissues, bleeding occurred. This patient rejects further therapy.

In one case with mediastinal lymphadenopathy and multifocal pulmonary nodal infiltrations, diagnostic videomediastinoscopy was followed by treatment with thalidomide with a good response. When the therapy was tapered, progression was noted in control CT, and thalidomide was re-entered.

In the MCD group, four patients died. Two deaths occurred due to multiorgan failure related to $\mathrm{CD}$ and complication after anti-CD therapy. Two patients died in the course of cardiac disorder.

One patient with a smoking history had primary lung cancer and underwent thoracotomy with upper left lobectomy and lymphadenectomy.

\section{Discussion}

Despite the rarity of CD, we have accumulated a set of diverse cases that enabled a comprehensive review of the diagnosis and therapeutic options available for this disorder. Furthermore, this study demonstrates the significance of including CD in the differential diagnosis of other diseases because it continues to earn its reputation as a "clinical mimicker" and is often (especially with the UCD variant) misdiagnosed or undiagnosed (1,2,36-38). After 

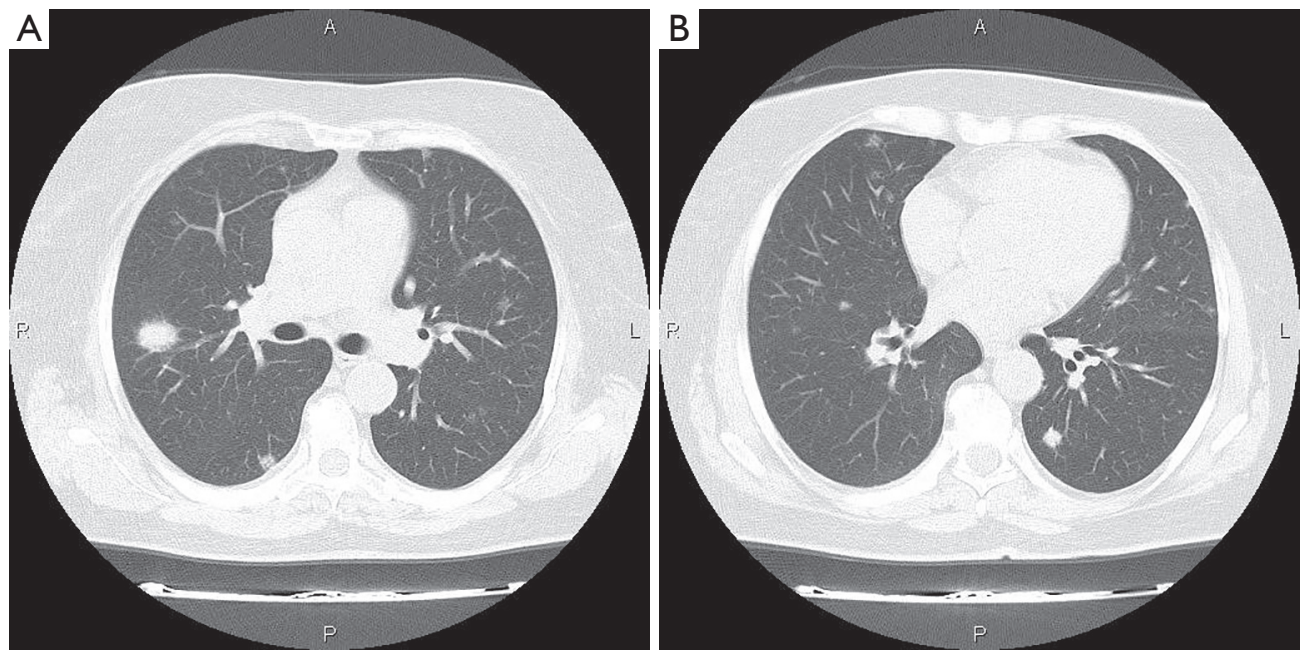

Figure 7 Chest CT shows bilaterally isolated lung tumors (A,B).

histopathological examination, which remains the only method of obtaining a certain diagnosis of this disease, we identified all three histological types of CD among our cases. In this regard, our findings are compatible with those of most other reports, with the HV type occurring most frequently, the PC type following, and the mixed type the least frequent $(2,6,8,19,36)$. The locations of the tumor and diffusive changes, including the cervix, thorax, axilla, and abdomen are also concordant with available literature $(8,30,36)$. The isolated intrapulmonary type of CD is quite rare $(39,40)$. Three patients in the current series had CD that involved isolated diffuse lung parenchyma, two cases presented mixed cellularity type, and one was of PC type (Figure 7).

The most common type of CD in our study group, HV, was identified in most of the possible locations found in other case reports, confirming that HV type CD has no limits regarding localization $(22,26,30)$. Most commonly, it was accompanied by local lymphadenopathy. In two cases of $\mathrm{CD}$, generalized pathological lymph node enlargement was observed. Earlier publications suggested that in cases of $\mathrm{HV}$ type CD, complete surgical resection of the mass (with proximal lymph nodes, if affected) is the gold standard $(8,24,41-44)$. In our study group, this resolution was also the most common-but not the sole-approach. In one case, radiotherapy was also performed. We described one patient who underwent partial excision because of surgical difficulties that made complete resection impossible, and despite the patient's declining further therapy, he continued to live without signs of progression. One of our patients with HV type CD received thalidomide-based pharmacotherapy and experienced regression but upon relapse with hypercytokinemia he died despite intensive treatment.

The next in terms of occurrence rate was PC type CD. At diagnosis, it was most commonly found as multifocal lymphadenopathy with no predominant mass, although a case of generalized lymphadenopathy and one involving a localized tumor accompanied by affected lymph nodes were also noted. Together with pronounced, unspecific symptomatologic manifestations, they represented the typical clinical presentation of PC type CD $(3,31,33,36,42)$. Because of its typical multiple, non-localized lymph node effects, a complete resection is seldom an option other than for purely diagnostic purposes $(35,42)$. Thus, a pharmacological approach is recommended as described in the therapeutic section of this review $(18,19,30,35,42,45,46)$. However, in our cases, we used both pharmaceutical and surgery-based treatments. For pharmacotherapy, thalidomide-based monotherapy was the most common approach; as we noted, there was one case of regression and one case of remission with no relapse, and in both cases, thalidomide was continued as maintenance therapy. One patient who retrospectively was identified as being HHV-8-positive, responded to prednisone, but the followup was quite short. A combined therapy (Cy, thalidomide, prednisone) was administered to one patient whose case, in retrospective analysis, might have been MCD-like because of associating autoimmune disease. This patient was quite symptomatic and died of multiorgan failure and infectious complications after anti-CD therapy. Other authors also perceive multidrug therapy as the treatment of last resort 
in PC type MCD and still note unsatisfactory results with prognosis remaining "poor" $(2,35,36,45)$. However, access to novel anti-IL-6 therapies could have changed the course of the disease in this patient.

Two patients with unicentric PC type CD underwent surgical excision as the initial therapy. One remained in remission for more than 11 years, and after the second excision relapsed locally within 7 years and underwent another surgical procedure. In case of PC type CD (typically an MCD variant), the literature most often mentions surgery only as a means of providing temporary, symptomatic relief and obtaining material for histopathological examination, not as a primary therapeutic option $(42,43,45,47)$.

In our case series, only two cases of mixed CD were identified. Both patients underwent exploratory thoracotomy. Because of isolated, multiple tumors in both lungs, they underwent surgery to yield material for histopathological examination. After the diagnosis of MC type, MCD was established, these patients were referred to hematologists for further treatment. Other authors infrequently have described this type in their case reports $(2,36)$. However, we also demonstrated that the features of $\mathrm{HV}$ and PC in MCD often coexist to some extent.

Mixed CD gives the clinician the broadest choice of approach. In the case of our two patients with an isolated localized mass in the lungs of unknown etiology, we opted for surgery. Both patients had full diagnostic procedures in the pulmonology department, but the findings did not settle the etiology of the lung tumors. One patient even had a cryo-biopsy, but the final histopathology result resulted as chronic inflammation. In both cases, the complete surgical resection during the surgery was impossible, and the procedure had to be limited to a diagnostic, partial excision. In the case of mixed type $\mathrm{CD}$, the literature does not offer a clear gold standard therapy, probably because of limited data regarding this type.

In our case-based analysis, the different therapeutic approach to different CD subtypes was rather maintained (48). The best results were achieved with surgical treatment of HV type CD and pharmacotherapy for PC type CD (46). Surgery remains the primary method of obtaining material for histopathological examination for both types and is a treatment providing relief in PC type CD $(42,43,45,47)$. The accuracy of the histopathological examination is essential and should follow the current diagnostic criteria $(49,50)$. Regarding the pharmacotherapy, it is important to follow the novel recommendations, including anti-IL-6 treatment. In the case of MCD, the disease activity and staging must be assessed, and treatment should be tailored to fit the disease type and its severity.

\section{Acknowledgments}

None.

\section{Footnote}

Conflicts of Interest: The authors have no conflicts of interest to declare.

Ethical Statement: The authors are accountable for all aspects of the work in ensuring that questions related to the accuracy or integrity of any part of the work are appropriately investigated and resolved. Informed patient's consent was obtained.

\section{References}

1. Takhar RP. Intrathoracic Castleman's disease: "An important clinical mimicker". Lung India 2017;34:197-99.

2. Wu D, Lim MS, Jaffe ES. Pathology of Castleman Disease. Hematol Oncol Clin North Am 2018;32:37-52.

3. Wong RSM. Unicentric Castleman Disease. Hematol Oncol Clin North Am 2018;32:65-73.

4. Cervantes CE, Correa R. Castleman Disease: A Rare Condition with Endocrine Manifestations. Cureus 2015;7:e380.

5. Tanaka O, Kiryu T, Hirose Y, et al. Chest wall Castleman's disease: CT and MRI findings. Radiat Med 2006;24:529-33.

6. Barua A, Vachlas K, Milton R, et al. Castleman's disease a diagnostic dilemma. J Cardiothorac Surg 2014;9:170.

7. Yoshizaki K, Murayama S, Ito H, et al. The Role of Interleukin-6 in Castleman Disease. Hematol Oncol Clin North Am 2018;32:23-36.

8. Fajgenbaum DC, Uldrick TS, Bagg A, et al. International, evidence-based consensus diagnostic criteria for HHV-8negative/idiopathic multicentric Castleman disease. Blood 2017;129:1646-57.

9. Goldblum JR, Lamps LW, McKenney JK, et al. Rosai and Ackerman's surgical pathology. 11 ed. Philadelphia: Elsevier; 2018.

10. Minemura H, Tanino Y, Ikeda K. Possible Association of Multicentric Castleman's Disease with Autoimmune Lymphoproliferative Syndrome. Biores Open Access 2018;7:47-51. 
11. Rollins-Raval MA, Marafioti T, Swerdlow SH, et al. The number and growth pattern of plasmacytoid dendritic cells vary in different types of reactive lymph nodes: an immunohistochemical study. Hum Pathol 2013;44:1003-10.

12. Izumi M, Mochizuki M, Kuroda M, et al. Angiomyoid proliferative lesion: an unusual stroma-rich variant of Castleman's disease of hyaline-vascular type. Virchows Archiv 2002;441:400-5.

13. Chan ACL, Chan KW, Chan JKC, et al. Development of follicular dendritic cell sarcoma in hyaline-vascular Castleman's disease of the nasopharynx: tracing its evolution by sequential biopsies. Histopathology 2001;38:510-8.

14. Jegalian AG, Facchetti F, Jaffe ES. Plasmacytoid dendritic cells: physiologic roles and pathologic states. Adv Anat Pathol 2009;16:392-404.

15. Siddiqi IN, Brynes RK, Wang E. B-cell lymphoma with hyaline vascular Castleman disease-like features. A clinicopathologic study. Am J Clin Pathol 2011;135:901-14.

16. Chittal SM, Caveriviére P, Voigt JJ, et al. Follicular lymphoma with abundant PAS-positive extracellular material. Immunohistochemical and ultrastructural observations. Am J Surg Pathol 1987;11:618-24.

17. Xie Y, Vallangeon B, Liu X, et al. Plasmacytic or lymphoplasmacytic infiltrate in lymph nodes: Diagnostic approach and differential considerations. Indian J Pathol Microbiol 2016;59:446-56.

18. van Rhee F, Voorhees P, Dispenzieri A, et al. International, evidence-based consensus treatment guidelines for idiopathic multicentric Castleman disease. Blood 2018;132:2115-24.

19. Chan KL, Lade S, Prince HM, et al. Update and new approaches in the treatment of Castleman disease. J Blood Med 2016;7:145-58.

20. Fajgenbaum DC. Novel insight and therapeutic approaches in idiopathic multicentric Castleman disease. Blood 2018;132:2323-30.

21. Legras A, Tallet A, Didelot A, et al. Clinical and molecular characteristics of unicentric mediastinal Castleman disease. J Thorac Dis 2018;10:2079-88.

22. Oksenhendler E, Boutboul D, Fajgenbaum D, et al. The full spectrum of Castleman disease: 273 patients studied over 20 years. Br J Haematol 2018;180:206-16.

23. Haager B, Kayser G, Schmid S, et al. Intrapulmonary Castleman's disease pretending to be a lung cancer-work up of an intrapulmonary tumour. Ann Thorac Cardiovasc Surg 2016;22:258-60.

24. van Rhee F, Stone K, Szmania S, et al. Castleman disease in the 21st century: an update on diagnosis, assessment, and therapy. Clin Adv Hematol Oncol 2010;8:486-98.

25. Akiyama $M$, Yasuoka $H$, Takeuchi T. Interleukin-6 in idiopathic multicentric Castleman's disease after long-term tocilizumab. Ann Hematol 2017;96:2117-9.

26. Bower M. How I treat HIV-associated multicentric Castleman disease. Blood 2010;116:4415-21.

27. Miranda RN, Khoury JD, Medeiros LJ. Multicentric Castleman Disease in: Atlas of Lymph Node Pathology. New York: Springer New York; 2013, 111-3.

28. Fajgenbaum DC, Shilling D. Castleman Disease Pathogenesis. Hematol Oncol Clin North Am 2018;32:11-21.

29. Simpson D. Epidemiology of Castleman disease. Hematol Oncol Clin North Am 2018;32:1-10.

30. van Rhee F, Greenway A, Stone K. Treatment of Idiopathic Castleman Disease. Hematol Oncol Clin North Am 2018;32:89-106.

31. Dispenzieri A. How I treat POEMS syndrome. Blood 2012;119:5650-8.

32. Sarana B, Jaal J, Tamm H, et al. Resection of unicentric interlobar Castleman disease with following adjuvant radiotherapy. SAGE Open Med Case Rep 2017;5:2050313X17744481.

33. Gérard L, Michot JM, Burcheri S, et al. Rituximab decreases the risk of lymphoma in patients with HIVassociated multicentric Castleman disease. Blood 2012;119:2228-33.

34. Nagao A, Nakazawa S, Hanabusa H. Short-term efficacy of the IL6 receptor antibody tocilizumab in patients with $\mathrm{HIV}$-associated multicentric Castleman disease: report of two cases. J Hematol Oncol 2014;7:10.

35. Fajgenbaum DC, Kurzrock R. Siltuximab: a targeted therapy for idiopathic multicentric Castleman disease. Immunotherapy 2016;8:17-26.

36. Szalat R, Munshi NC. Diagnosis of Castleman Disease. Hematol Oncol Clin North Am 2018;32:53-64.

37. Tampakis A, Tampaki EC, Daikeler T. Intrathoracic tumor of the chest wall: A case of Castleman's disease mimicking myositis of the lower extremities. Gen Thorac Cardiovasc Surg 2017;65:664-6.

38. Horio H, Hijima T, Sakaguchi K, et al. Mediastinal Castleman disease associated with pulmonary carcinoma, mimicking N2 stage lung cancer. Jpn J Thorac Cardiovasc Surg 2005;53:286-9.

39. Racil H, Rouhou SC, Ismail O, et al. Castleman's Disease: An Intrapulmonary Form with Intrafissural Development. ScientificWorldJournal 2009;9:940-5. 
40. Huang H, Feng R, Li J, et al. Castleman disease-associated diffuse parenchymal lung disease. A STROBE-compliant retrospective observational analysis of 22 cases in a tertiary Chinese hospital. Medicine 2017;96:e8173.

41. Gunluoglu G, Olcmen A, Sokucu SN, et al. Intrapulmonary-located Castleman's Disease, Which Was Surgically Resected without Pulmonary Resection. Ann Thorac Cardiovasc Surg 2011;17:580-3.

42. Testori A, Voulaz E, Alloisio M, et al. Multicentric castleman's disease resembling metastatic lung carcinoma. A case report. Clin Case Rep 2018;6:473-5.

43. Wala SJ, Fallon EM, Forlenza CJ, et al. Unicentric Castleman disease in the mediastinum. J Pediatr Surg Case Rep 2018;34:51-3.

44. Mitsos S, Stamatopoulos A, Patrini D, et al. The role of surgical resection in Unicentric Castleman's disease: a systematic review. Adv Respir Med 2018;86:36-43.

45. Caroline Ribeiro Sales A, Romão de Souza Junior V, Iglis de Oliveira M, et al. Multicentric Castleman's disease in

Cite this article as: Wojtyś M, Piekarska A, Kunc M, Ptaszyński K, Biernat W, Zaucha JM, Waloszczyk P, Lisowski P, Kubisa B, Grodzki T. Clinicopathological comparison and therapeutic approach to Castleman disease-a case-based review. J Thorac Dis 2019;11(11):4859-4874. doi: 10.21037/ jtd.2019.10.73 human immunodeficiency virus infection: two case reports. J Med Case Rep 2018;12:117.

46. Cao W, Liang S, Liu J, et al. Castleman's disease presenting in the lungs: A report of two cases. Oncol Lett 2015;10:1041-3.

47. Lurain K, Yarchoan R, Uldrick TS. Treatment of Kaposi Sarcoma Herpesvirus - Associated Multicentric Castleman Disease. Hematol Oncol Clin North Am 2018;32:75-88.

48. Erdogan A, Eser İ, Özbilim G. Posterior mediastinal localization of Castleman's disease: report of a case. Surg Today 2004;34:772-3.

49. Nadir A, Colak N, Koktener A, et al. Isolated Intrapulmonary Castleman's disease: a case report, review of the literature. Ann Thorac Cardiovasc Surg 2014;20 Suppl:689-91.

50. Hountis P, Dedeilias P, Douzinas M. The management of Castleman's disease of the mediastinum: a case report. Cases J 2008;1:330. 